\title{
Robustness of topologically protected transport in graphene-boron nitride lateral heterostructures
}

\author{
D S L Abergel \\ Nordita, KTH Royal Institute of Technology and Stockholm University, \\ Roslagstullsbacken 23, SE-106 91 Stockholm, Sweden. \\ Center for Quantum Materials, KTH and Nordita, Roslagstullsbacken 11, SE-106 91 \\ Stockholm, Sweden.
}

\begin{abstract}
Previously, graphene nanoribbons set in lateral heterostructures with hexagonal boron nitride were predicted to support topologically protected states at low energy. We investigate how robust the transport properties of these states are against lattice disorder. We find that forms of disorder that do not couple the two valleys of the zigzag graphene nanoribbon do not impact the transport properties at low bias, indicating that these lateral heterostructures are very promising candidates for chipscale conducting interconnects. Forms of disorder that do couple the two valleys, such as vacancies in the graphene ribbon, or substantial inclusions of armchair edges at the graphene-hexagonal boron nitride interface will negatively affect the transport. However, these forms of disorder are not commonly seen in current experiments.
\end{abstract}




\section{Introduction}

If graphene devices are to be integrated into circuits with multiple components, it will be highly convenient to have graphene connectors to allow electronic current to move between different devices. Zigzag graphene nanoribbons (ZZGNRs) are known to host metallic edge states, and are attractive candidates for such current-carrying wires. This idea has been investigated previously, but it was found that the transport properties of the ZZGNRs were highly fragile against edge roughness [1, 2, 3, 4, 5]. This is a crucial issue, since any growth-based fabrication method for will necessarily introduce lattice scale disorder into the ZZGNRs. Lateral heterostructures are monolayers where two or more 2D materials are 'stitched' together to form 1D interfaces. It is possible to grow lateral heterostructures of graphene and insulating hexagonal boron nitride (hBN) [6, 17, 8, 9, 10, 11, 12, and much theoretical work, especially using ab initio methods, has been done to investigate their electronic properties [13, 14, 15, 16, 17]. However, the topological properties of these lateral heterostructures has received only very limited attention [18]. Jung et al. suggested that by engineering hBN "cladding" on either side of the ZZGNR, then the topological properties of the combined system can ensure that there are always conducting channels in the graphene [18]. This topological protection is akin to the Jackiw-Rebbi states that are predicted to exist at mass-inversion boundaries in hexagonal crystals [19], and is described by a valley Chern number [18, 20].

The atomic-scale precision required to produce these lateral heterostructures does currently exist [11, 12], but the graphene-hBN interfaces defined by such techniques are still rather disordered. This issue is crucial in the context of topological protection because, as explained in Refs. [18, 20] the topological protection described by the valley Chern number only persists as long as the two valleys in the graphene are not coupled to each other. However, the edge roughness may constitute short-range disorder that is strong enough to scatter electrons between the two valleys, breaking the topological protection and allowing backscattering which reduces the conductance.

In this manuscript, we present a full analysis of the role of atomic-scale disorder on the transport properties of ZZGNR-hBN lateral heterostructures. We find that only certain types of lattice disorder break the topological protection and couple the valleys, allowing the backscattering. Specifically, inclusion of substantial regions of armchair interface and graphene vacancies will do this, but rough edges and inclusions of random boron or nitrogen atoms in the graphene ribbon will not. We contend that the explanation for this is that the latter types of disorder are smooth enough in the sense that they can be reached from the original Hamiltonian by an adiabatic transformation, and therefore they do not modify the overall topological properties of the system and hence do not couple the valleys. This analysis, combined with recent advances in fabrication techniques, reopens the issue of ZZGNRs clad with hBN as a highly suitable method of providing chip-scale conducting channels. 

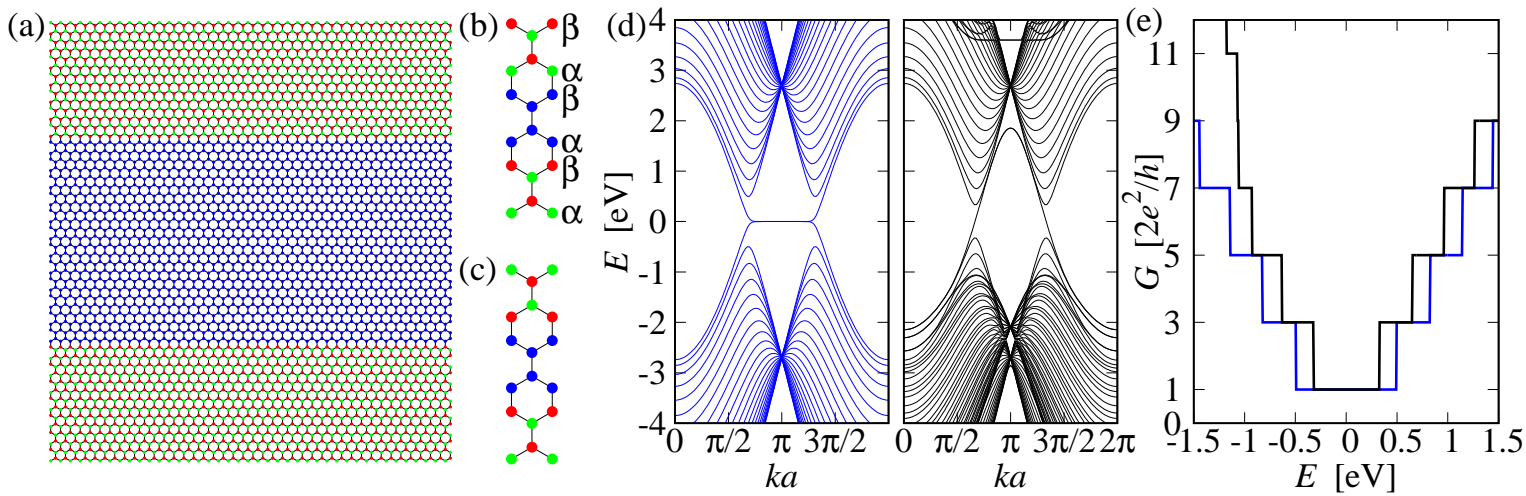

Figure 1. (a) The non-disordered ZZGNR with hBN cladding. Atomic sites are colour-coded as follows. Carbon: blue; nitrogen: red; boron: green. (b) Sketch of the 'same' topology configuration. Notice that the $\alpha$ site both above and below the ZZGNR hosts a boron atom. (c) Sketch of the 'opposite' topology configuration. In this case, the $\alpha$ site below the ZZGNR hosts a boron atom, while above the ZZGNR, the $\alpha$ site hosts a nitrogen atom. (d) Band structures of non-disordered 5nm ZZGNR without (blue) and with (black) hBN cladding. (e) Conductance of non-disordered 5nm ZZGNR without (blue) and with (black) hBN cladding.

\section{Methods}

To demonstrate the robustness of the predicted low bias transport channel, we compute the 1D charge conductance using the Landauer-Buttiker scattering formalism within the Kwant package [21]. We use a ZZGNR with width $5.0 \mathrm{~nm}$ and length $10.0 \mathrm{~nm}$, corresponding to chip-scale dimensions, and we take the hBN width to be $3.0 \mathrm{~nm}$, which is wide enough to ensure the topological properties are manifested whilst still being small enough to ensure reasonable computation time. This configuration is shown in Fig. 1(a). To compute the spectrum and wave functions in the leads and in the scattering region, we use a nearest-neighbour tight binding model. The tight binding parameterisation requires the onsite energies $U_{i}$ for each chemical species, and the hopping elements $t_{i j}$ between them, where $i, j \in\{\mathrm{C}, \mathrm{B}, \mathrm{N}\}$ denote the chemical species. Throughout, we use $U_{\mathrm{C}}=0, U_{\mathrm{B}}=3.6 \mathrm{eV}, U_{\mathrm{N}}=-1.0 \mathrm{eV}, t_{\mathrm{CC}}=2.7 \mathrm{eV}, t_{\mathrm{CB}}=2.1 \mathrm{eV}, t_{\mathrm{CN}}=2.3 \mathrm{eV}$, and $t_{\mathrm{BN}}=2.5 \mathrm{eV}[15]$. We stress that so long as $\operatorname{signum}\left(U_{\mathrm{N}}\right) \neq \operatorname{signum}\left(U_{\mathrm{B}}\right)$, the precise values of the tight binding parameters do not make any difference to the overall topological properties of the system, and merely give small quantitative changes to the band structure and hence the exact positions of the conductance steps.

In principle, a more accurate description of the band structure of the ribbons is given by a third-nearest neighbour tight binding theory [22, 23], but this additional complexity changes none of the qualitative features of the results, or the considerations about the topology of the system. Therefore, we restrict our discussion to the nearest neighbour model for clarity. In the Supplementary Material, we show data which justifies this assumption further. 

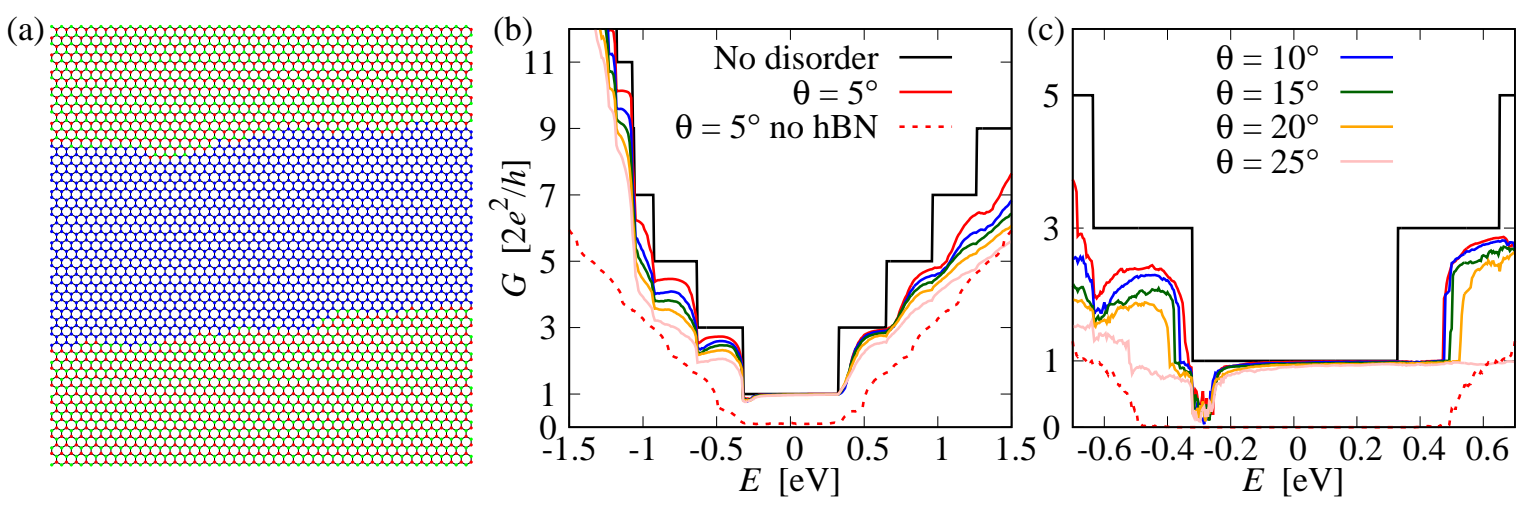

Figure 2. (a) Typical disordered wire with $\theta=15^{\circ}$. (b) Averaged conductance, and (c) fifth percentile conductance of 200 disordered wire realisations.

\section{Results and discussion}

Figure 1(a) shows the non-disordered ZZGNR with hBN cladding that forms the basis of the ribbons we consider in this manuscript. In the 'same' topology configuration, sketched in Fig. 1(b), the $\alpha$ and $\beta$ sublattices of the two regions of hBN cladding have the same chemical orientation. (Throughout, we use the notation $\alpha$ and $\beta$ for the two sublattices of the hexagonal crystal to avoid confusion between the 'B' sublattice and the chemical symbol for boron atoms.) In this case, there are no topologically protected states in the graphene because the mass gap generated in the hBN has the same sign in both $\mathrm{hBN}$ regions. However, when the chemical orientation is 'opposite', sketched in Fig. 1(a) and Fig. 1(c), the mass term has opposite sign in the two hBN regions, and therefore their valley Chern number is different. This is sufficient to ensure that there are topologically protected states in the graphene [18].

The band structures and conductance of the ZZGNR are shown in Figs. 1(d), (e), respectively, where the blue lines correspond to the ZZGNR with no cladding and the black lines are for cladding in the 'opposite' topology configuration with nitrogen termination. These are fully consistent with the results of Ref. [18. The units on the vertical axis are $2 e^{2} / h$ to account for the spin degeneracy of each of the bands. We see that the cladding causes the flat band edge states to be removed and instead there are dispersing modes at zero energy. These are the topologically protected Jackiw-Rebbilike modes, and they manifest in the conductance by a finite minimum conductance plateau $-0.35 \mathrm{eV}<E<0.35 \mathrm{eV}$. This is the feature that we are most interested in, since it defines the conducting channel that may be used to direct current between graphene devices. The sharp increase in the conductance at roughly $E=-1 \mathrm{eV}$ is caused by conduction through modes located mainly on the nitrogen atoms which can be seen in the band structure in Fig. 1(d). Modes located mainly on the boron atoms are located at approximately $E=3.6 \mathrm{eV}$ and so do not appear in the conductance plot. The presence of the cladding also slightly reduces the gap between the first non-topologically protected modes in the conduction and valence bands. 
Our central result is shown in Fig. 2, where we plot the conductance of ZZGNRs with random edge disorder and hBN cladding. To create disordered ribbons, we keep track of the two boundaries between the ZZGNR and the hBN. For each lattice unit cell along the length of the wire, we allow the $y$ coordinate of the boundaries to change relative to the previous unit cell $y_{i}=y_{i-1}+\delta y$ where $\delta y=a \tan \theta_{i}$ and $a$ is the lattice constant. The deflection angle $\theta_{i}$ is a random variable characterised by a Gaussian distribution with variance $\theta$, so that higher values of $\theta$ correspond to a higher propensity towards rough edges. Figure 2(a) shows a typical example ribbon with $\theta=15^{\circ}$, and we stress that the shape of the upper and lower boundaries are independent of each other. For each value of $\theta$ we compute the conductance of 200 ribbons with random disordered edges, calculate the mean conductance at each value of energy, and show the result in Fig. 2(b). For even high values of edge roughness, on average, the finite minimum conductance at low energy remains intact, indicating that the topologically protected modes in the graphene are resiliant against edge disorder. The reduction in the conductance at higher energy (i.e. away from the finite minimum conductance plateau) is caused by backscattering in the non-topologically protected states as would be expected in ZZGNRs without hBN cladding [3]. To confirm the effect of the cladding, the red dotted line shows the averaged conductance for the exact same 200 ZZGNRs with $\theta=5^{\circ}$ but with the hBN cladding removed, and therefore with no topological effects. In this case, the finite minimum conductance plateau at low energy is completely absent. In Fig. 2(c) we show the fifth percentile conductance at each energy, i.e. the level at which 190 of the 200 disorder realisations have better conductance than the line shown. This shows that for the vast majority of randomly disordered ZZGNRs, the finite minimum conductance plateau remains intact.

We now examine some more controlled forms of disorder to determine which contribute to the breakdown of the topological protection. Figure 3 shows the calculated conductance for carbon vacancies and boron substitution in the ZZGNR. In principle, since this type of disorder has the shortest associated length scale, it should scatter electrons between states separated by a momentum of the order of the Brillouin zone size, and therefore couple the $K$ and $K^{\prime}$ valleys the most strongly, leading to breakdown of the topological protection of the low energy modes. In Fig. 33(b), we show the conductance of our standard ZZGNR with a single carbon atom removed from the $\beta$ sublattice of a unit cell near the lower edge. The location of the vacancies are shown in panel (a), where the colour of the dot corresponds to the colour of the line in the conductance plot. If it is removed from the first unit cell (blue line), then a sharp decrease in the conductance at the top of the low energy finite conductance plateau is seen. As the vacancy is moved into the ZZGNR (red, green, and orange lines) the position of the resonance moves towards zero energy. When the vacancy is in the center of the ZZGNR (black line), the conductance dip is at the center of the plateau. This variation in the energy at which the conductance dip occurs with the position of the vacancy is related to the change in the wave function with energy, since modes with energy near the edge of the finite minimum conductance plateau are localised near the edges of the ZZGNR. The 
(a)

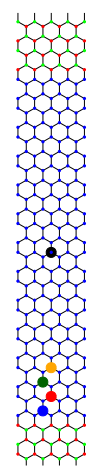

(b)

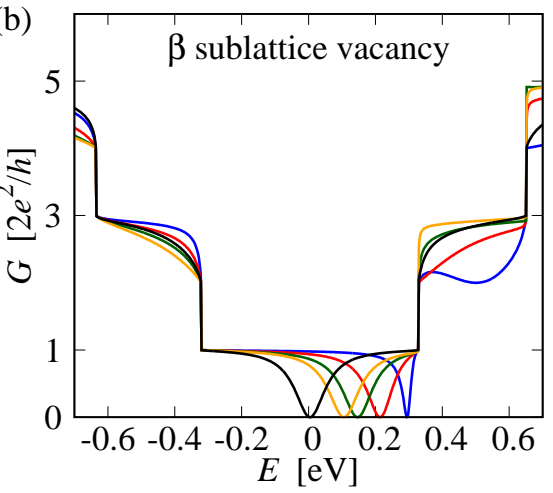

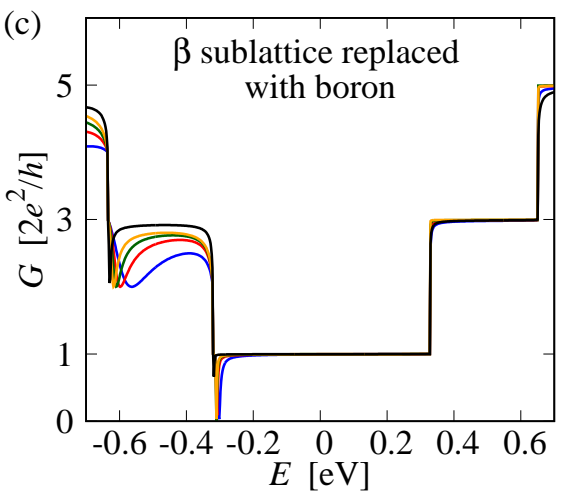

Figure 3. Single site disorder. (a) Sketch of hBN-clad ZZGNR showing the position of the vacancy or substitution site by dots corresponding in color to the lines in (b) and (c). (b) Conductance with graphene vacancy on the $\beta$ sublattice. (c) Conductance with boron substitution on the $\beta$ sublattice.
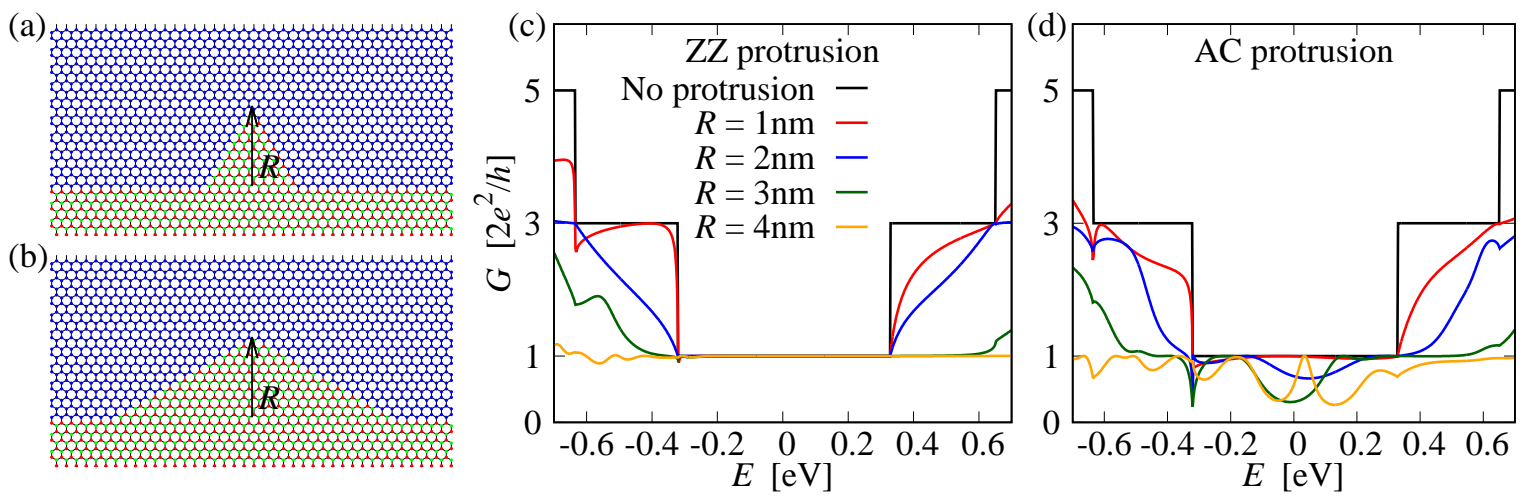

Figure 4. Protrusions with ordered edges. (a) Sketch of a zigzag protrusion. (b) Sketch of an armchair protrusion. (c) Conductance of hBN-clad ZZGNR with zigzag protrusion. (d) Conductance of hBN-clad ZZGNR with armchair protrusion.

mirror symmetry of the opposite topology configuration insists that vacancies on the $\alpha$ sublattice at the upper edge have their conductance dip at the same energy. Swapping either the sublattice or the edge makes the sign of the energy at which the conductance dip occurs change.

In contrast, Fig. 3(c) shows that substitution of a carbon atom with a boron atom has very little effect on the conductance. We have verified that nitrogen substitution gives even smaller changes to the conductance. This explains the small dip in the conductance in Fig. 2 at the low energy side of the finite minimum conductance plateau, since mild edge roughness is replicated by many boron or nitrogen substitutions.

In Fig. 4 we demonstrate the crucial difference between zigzag and armchair protrusions of $\mathrm{hBN}$ into the ZZGNR. It is well known that armchair edges strongly couple the two valleys, since the projection of the 2D Brillouin zone of bulk graphene onto the 1D Brillouin zone of a ribbon in the armchair direction projects the $K$ and $K^{\prime}$ valleys to the same point. In contrast, the zigzag ribbon projects the $K$ and $K^{\prime}$ valleys to 
different points in the 1D Brillouin zone and so the two valleys are not strongly coupled (see the Supplementary Material). Figures 4(a) and (b) show a triangular protrusion of hBN into the ZZGNR with width $R$ with zigzag and armchair edges, respectively. Figure 4(c) shows the calculated conductance for the zigzag protrusion up to $R=4 \mathrm{~nm}$ (i.e. $80 \%$ of the ZZGNR width). The finite conductance minimum associated with the topological states is perfectly intact for all zigzag protrusions, indicating that there is no backscattering induced in this case. However, backscattering is induced in the high energy non-topologically protected states by the zigzag protrusion. Figure 4(d) shows the equivalent data for armchair protrusions. In this case, the valley coupling manifests as substantial and apparently uncontrolled oscillations in the conductance as a function of energy in the finite minimum conductance plateau. This indicates that substantial inclusions of armchair edges in the ZZGNR lead to sub-optimal low energy transport properties.

\section{Conclusions}

We have shown highly promising results for the use of hBN-clad ZZGNRs as chip-scale interconnects with perfect ballistic conductance. In particular, we have systematically investigated the impact of lattice disorder of various types on the transport properties of such ZZGNRs. Our conclusion is that only atomic-scale disorder which strongly couples the $K$ and $K^{\prime}$ valley of the bulk graphene - thus invalidating the valley Chern number construction and hence lifting the topological protection of the low energy modes - is effective at inducing backscattering between the topological states and weakening the transport. Other types of disorder, such as edge roughness and chemical substitution, do not impact the transport. This is because, at least within a tight binding model, atomic substitution amounts only to changing the onsite energy and hopping elements by a finite amount. This is a only a small change in the theory from the 'clean' case, or, in the language of topology, it is an adiabatic transformation and hence retains the same topological properties. This is a very encouraging result, since zigzag interfaces are energetically favourable to armchair interfaces, and have been shown to dominate in fabrication by a ratio of better than $3: 1$, and carbon vacancies are very rare [11]. However, devices do exhibit substantial edge roughness. Therefore, topologically protected transport may be expected in such devices even with current growth techniques.

One issue that has so far not been addressed is that of the $1.8 \%$ lattice mismatch between graphene and hBN. However, the results presented here are enough to justify that this should not present a problem for short interconnects. For ZZGNRs which are less than approximately 50 unit cells in length (NB, the one we have modelled here is 40 unit cells long), the strain buildup in the hBN due to the mismatch should result only in the modification of the hopping parameters between the last few rows of BN lattice sites and the first few rows of carbon lattice sites. However, our results indicate that small modifications in the hopping parameters do not result in changes in the topological 
properties of the system and hence there should be no impact on the topologically protected transport channels. For longer wires, we have explicitely calculated the conductance with a missing nitrogen atom at the edge (see Supplementary Material), and found that there was no impact on the topological modes in the graphene because there is essentially no wave function weight in the hBN for energies $U_{\mathrm{N}}<E<U_{\mathrm{B}}$. Hence, the strain induced by the lattice mismatch for chip-scale ZZGNRs should have minimal impact on the transport properties.

\section{Acknowlegements}

This work is supported by Nordita and by ERC project DM-321031.

\section{References}

[1] Gunlycke D, Areshkin D A and White C T 2007 Appl. Phys. Lett. 90142104

[2] Yoon Y and Guo J 2007 Appl. Phys. Lett. 91073103

[3] Basu D, Gilbert M J, Register L F, Banerjee S K and MacDonald A H 2008 Appl. Phys. Lett. 92 042114

[4] Cresti A, Nemec N, Biel B, Niebler G, Triozon F, Cuniberti G and Roche S 2008 Nano Res. 1361

[5] Martin I and Blanter Y 2009 Phys. Rev. B 79235132

[6] Levendorf M P, Kim C J, Brown L, Huang P Y, Havener R W, Muller D A and Park J 2012 Nature 488627

[7] Sutter P, Cortes R, Lahiri J and Sutter E 2012 Nano Lett. 124869

[8] Liu Z, Ma L, Shi G, Zhou W, Gong Y, Lei S, Yang X, Zhang J, Yu J, Hackenberg K P, Babakhani A, Idrobo J C, Vagtai R, Lou J and Ajayan P M 2013 Nature Nanotech. 8119

[9] Liu L, Park J, Siegel D A, McCarty K F, Clark K W, Deng W, Basile L, Idrobo J C, Li A P and Gu G 2014 Science 343163

[10] Han G H, Rodríguez-Manzo J A, Lee C W, Kybert N J, Lerner M B, Qi Z J, Dattoli E N, Rappe A M, Drndic M and Johnson A T C 2013 ACS Nano 710129

[11] Gao Y, Zhang Y, Chen P, Li Y, Liu M, Gao T, Ma D, Chen Y, Cheng Z, Qiu X, Duan W and Liu Z 2013 Nano Lett. 133439

[12] Drost R, Uppstu A, Schulz F, Hmlinen S K, Ervasti M, Harju A and Liljeroth P 2014 Nano Lett. 145128

[13] Ding Y, Wang Y and Ni J 2009 Appl. Phys. Lett. 95123105

[14] Bhowmick S, Singh A K and Yakobson B I 2011 J. Phys. Chem. C 1159889

[15] Jungthawan S, Limpijumnong S and Kuo J L 2011 Phys. Rev. B 84235424

[16] Seol G and Guo J 2011 Appl. Phys. Lett. 98143107

[17] Li Y and Mazzarello R 2013 Phys. Rev. B 88045317

[18] Jung J, Qiao Z, Niu Q and MacDonald A H 2012 Nano Lett. 122936

[19] Abergel D S L, Edge J M and Balatsky A V 2014 New J. Phys. 16065012

[20] Li J, Morpurgo A F, Büttiker M and Martin I 2010 Phys. Rev. B 82245404

[21] Groth C W, Wimmer M, Akhmerov A R and Waintal X 2014 New J. Phys. 16063065

[22] Hancock Y, Uppstu A, Saloriutta K, Harju A and Puska M J 2010 Phys. Rev. B 81245402

[23] Wu Y and Childs P A 2010 Nanoscale Research Letters 662 


\title{
Supplementary material to 'Robustness of topologically protected transport in graphene- $\mathrm{hBN}$ heterostructures'
}

\author{
D S L Abergel \\ Nordita, KTH Royal Institute of Technology and Stockholm University, \\ Roslagstullsbacken 23, SE-106 91 Stockholm, Sweden. \\ Center for Quantum Materials, KTH and Nordita, Roslagstullsbacken 11, SE-106 91 \\ Stockholm, Sweden.
}



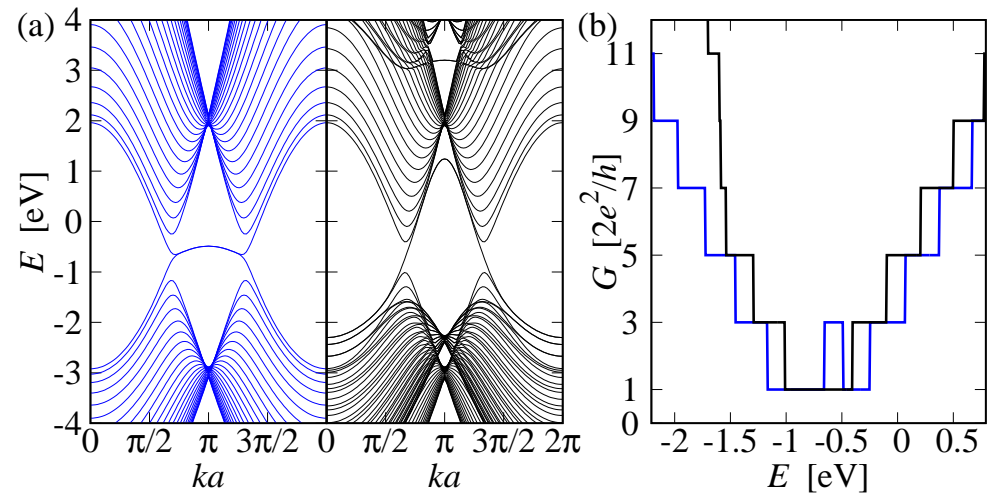

Figure 1. Equivalent data to Figure 1 of the main text for a third-nearest neighbour tight binding model.

\section{Third nearest neighbour tight binding model}

In principle a third-nearest neighbour (3NN) tight binding model is more accurate than the simple nearest neighbour model we use in the main text [22, 23]. Below, we provide justification for this assumption by comparing tight binding calculations for ZZGNRhBN lateral heterostructures.

To construct a $3 \mathrm{NN}$ model, successive neighbour hops are given by the formula

$$
t_{i j}(R)=A_{i j} e^{-R / \xi}
$$

where $i, j \in\{\mathrm{C}, \mathrm{B}, \mathrm{N}\}$ denote the the atomic species, $\xi=0.4329$ is the decay constant, and we calculate $A_{i j}$ from the nearest-neighbour hops which are taken from Ref. [15]. We emphasize that the precise values of the hopping parameters does not have a qualitative effect on the band structures or topology.

In Fig. 1, we show the band structure and conductance of a clean ZZGNR with $\mathrm{hBN}$ cladding in the $3 \mathrm{NN}$ tight binding approximation. The inclusion of the secondnearest neighbour terms are especially important since they introduce an effective onsite element. This includes a constant term which gives a rigid shift of the bands relative to the nominal zero of the energy axis. This is not physically meaningful. It also contains a contribution proportional to $k^{2}$ which introduces curvature to the previously flat band edge states when the $\mathrm{hBN}$ cladding is absent. This leads to an additional step to $G=6 e^{2} / h$ at $E \approx 0.5 \mathrm{eV}$ in the finite minimum conductance plateau.

When the hBN cladding is present, the edge states disappear and are replaced by the dispersing topologically protected states, just as in the nearest-neighbour model. The conductance plot for the 3NN case, shown in Fig. 1(b), demonstrates that the conductance with the hBN cladding is almost identical to that of the nearest-neighbour case once the energy shift is accounted for. This justifies our assumption to limit the main text to the nearest neighbour model for clarity.

In Fig. 2 we plot the conductance data for the 3NN model that corresponds to Fig. 2 of the main text. We stress that the exact same 200 disorder realisations were used in both cases and the only difference between the calculations is the number of 

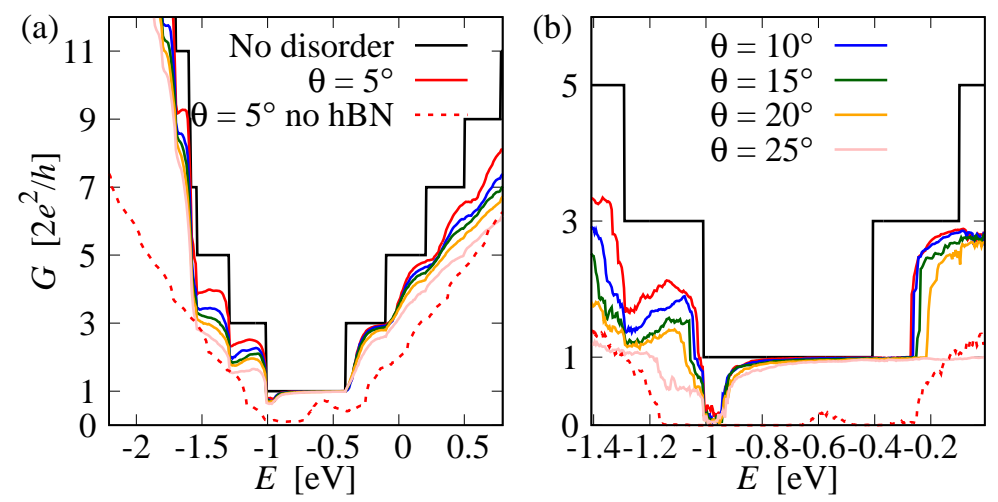

Figure 2. Equivalent data to Figure 2 of the main text for a third-nearest neighbour tight binding model.

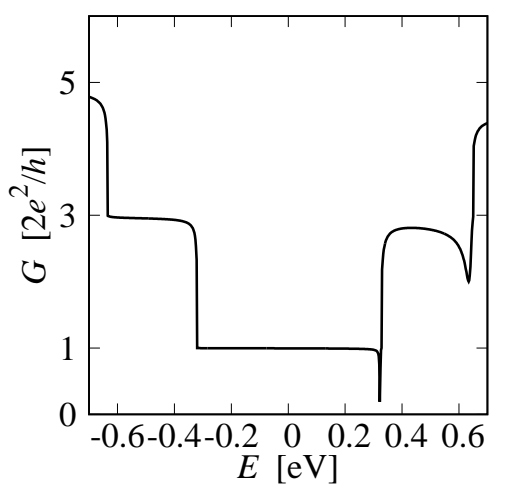

Figure 3. Conductance with nitrogen atom removed from ZZGNR-hBN interface to simulate the effect of strain relaxation in longer ribbons.

hopping terms in the tight binding theory. The qualitative features of the conductance in the $3 \mathrm{NN}$ case are identical to the nearest-neighbour approximation and all conclusions remain unchanged.

\section{Nitrogen vacancy at ZZGNR-hBN interface}

Figure 3 shows the conductance of a ZZGNR-hBN lateral heterostructure where one of the nitrogen sites in the terminating row of $\mathrm{hBN}$ has been removed. In conjuction with the observation that 'adiabatic' changes in the parameters of the hopping theory do not introduce backscattering, the almost perfect finite minimum conductance plateau in the figure shows that relaxation of strain buildup by a missing nitrogen line does not negatively impact the transport in the topologically protected ZZGNR states. This is because, at the energy of relevance, the wave function of the topologically protected states is located in the graphene and not on the boron or nitrogen atoms. 


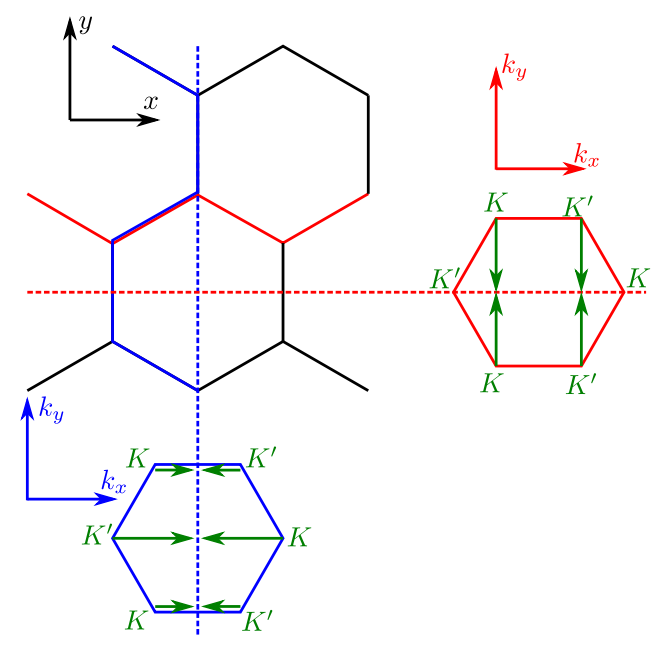

Figure 4. Projections of the 2D Brillouin zone into 1D for zigzag ribbons (red) and armchair ribbons (blue).

\section{One-dimensional Brillouin zones}

Figure 4 illustrates why armchair edges couple the two valleys in graphene nanoribbons, while zigzag edges do not. The hexagonal graphene lattice (shown in black, upper left) has a 2D Brillouin zone (BZ). When a ribbon is formed, the 1D translation symmetry manifests as a 1D BZ, which can be formed from the 2D BZ by projecting all momentum states down to the 1D BZ in the appropriate way.

For a zigzag edge (shown by the red line), the $x$ direction retains its translational symmetry, and so $k_{x}$ is still a good quantum number. Therefore, all $k_{y}$ states are projected down to $k_{y}=0$, as shown by the green arrows on the red BZ sketched in the upper-right part of the figure. This projection brings the $K$ and $K^{\prime}$ points to different parts of the $1 \mathrm{D} \mathrm{BZ}$, and hence the valleys are not coupled.

In contrast, for an armchair edge (shown by the blue line), the $y$ direction retains its translational symmetry and so $k_{y}$ remains a good quantum number. The $k_{x}$ states are therefore brought to $k_{x}=0$, and as shown in the lower-left part of the figure, the $K$ and $K^{\prime}$ points arrive at the same point in the 1D BZ. This couples the valleys strongly, and invalidates the valley Chern number construction [20]. 